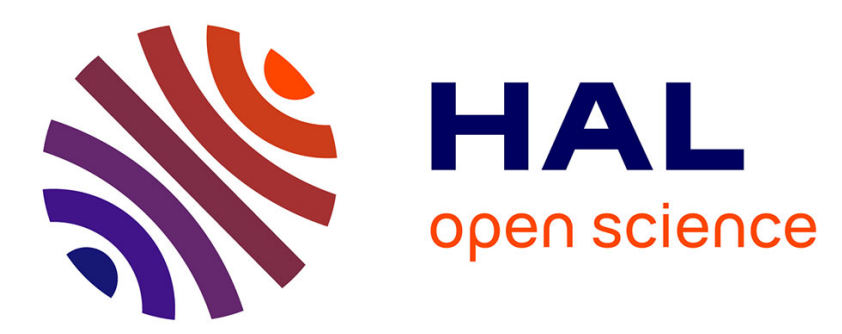

\title{
Dimensionnement et performances d'un stockage d'énergie thermique fluide/solides
}

\author{
J.L. Salagnac, J.A. Ayoub
}

\section{To cite this version:}

J.L. Salagnac, J.A. Ayoub. Dimensionnement et performances d'un stockage d'énergie thermique fluide/solides. Revue de Physique Appliquée, 1980, 15 (2), pp.213-218. 10.1051/rphysap:01980001502021300 . jpa-00244717

\section{HAL Id: jpa-00244717 https://hal.science/jpa-00244717}

Submitted on 1 Jan 1980

HAL is a multi-disciplinary open access archive for the deposit and dissemination of scientific research documents, whether they are published or not. The documents may come from teaching and research institutions in France or abroad, or from public or private research centers.
L'archive ouverte pluridisciplinaire HAL, est destinée au dépôt et à la diffusion de documents scientifiques de niveau recherche, publiés ou non, émanant des établissements d'enseignement et de recherche français ou étrangers, des laboratoires publics ou privés. 


\title{
Dimensionnement et performances d'un stockage d'énergie thermique fluide/solides
}

\author{
J. L. Salagnac et J. A. Ayoub \\ C.E.A. Saclay, Service d'Etudes Energétiques, B.P. 2, 91190 Gif sur Yvette, France
}

(Reçu le $1^{\text {er }}$ août 1979, révisé le 8 novembre 1979, accepté le 9 novembre 1979)

\begin{abstract}
Résumé. - Le stockage d'énergie thermique est un élément essentiel d'une boucle thermodynamique solaire. Tant que le niveau de température atteint dans une chaudière solaire à concentration reste inférieure à $300^{\circ} \mathrm{C}$ environ, on fait appel à des fluides organiques à point d'ébullition élevé comme milieu de stockage. Ces fluides étant coûteux, on étudie ici un moyen d'en réduire la quantité en les stockant dans les interstices d'un lit fixe d'agrégats de faible coût. Les dimensions et les performances d'un tel stockage sont évaluées et comparées à celles d'un stockage utilisant du fluide seul.
\end{abstract}

\begin{abstract}
Thermal energy storage is essential in thermodynamic solar power plants. As long as the temperature level attained in solar concentrators is less than about $300{ }^{\circ} \mathrm{C}$, the use of high temperature boiling point organic fluids is widespread. These fluids being expensive, we study in this paper a mean to reduce the amount of fluid by using a low cost bed of solids as a storage medium. The design and rating of such a packed bed are estimated and compared to those of a storage in fluid alone.
\end{abstract}

\begin{tabular}{ll}
\multicolumn{2}{c}{ Nomenclature } \\
\multicolumn{1}{c}{ DésIGNATION } \\
$a$ & \multicolumn{1}{c}{(} \\
$b$ & \\
$d$ & diamètre moyen des solides \\
$e$ & porosité \\
$h$ & coefficient d'échange \\
$l$ & longueur du stockage \\
$s$ & section du stockage \\
$t$ & temps \\
$v$ & vitesse interstitielle du fluid \\
$x$ & abscisse \\
$A$ & surface volumique de contact \\
& \multicolumn{1}{c}{$=\frac{6(1-e)}{d}$} \\
& chaleur spécifique \\
$C$ & diamètre \\
$D$ & température du fluide \\
$F(x, t)$ & puissance \\
$P$ & débit \\
$Q$ & température des solides \\
$S(x, t)$ & entropie \\
$S *$ & température de référence \\
$T_{0}$ & énergie interne \\
$U$ & volume \\
$V$ & travail \\
$W$ & abscisse sans dimension \\
$X$ &
\end{tabular}

\begin{tabular}{|c|c|c|c|}
\hline & $Z$ & temps sans dimension & \\
\hline UNITÉ & $\beta$ & & \\
\hline $\mathrm{s}^{-1}$ & $\begin{array}{l}o \\
\varepsilon_{1}\end{array}$ & $\begin{array}{l}\text { pourcentage } \\
\text { efficacité de récupération }\end{array}$ & \\
\hline & $\varepsilon_{2}$ & coefficient de récupération & \\
\hline $\mathrm{m}$ & $\begin{array}{l}\eta \\
\rho\end{array}$ & $\begin{array}{l}\text { rendement } \\
\text { masse volumique }\end{array}$ & $\mathrm{kg} \mathrm{m}^{-3}$ \\
\hline$\underset{\mathrm{m}^{2}}{\mathrm{~W} \mathrm{~m}^{-2} \mathrm{~K}^{-1}}$ & $\lambda$ & conductivité & $\mathrm{W} \mathrm{m}^{-1} \mathrm{~K}^{-1}$ \\
\hline $\begin{array}{c}\mathrm{s} \\
\mathrm{m} \cdot \mathrm{s}^{-1}\end{array}$ & & INDICES & \\
\hline $\begin{array}{c}\mathrm{m} \\
\mathrm{m}^{2} \mathrm{~m}^{-3}\end{array}$ & $\begin{array}{l}\mathrm{d} \\
\mathrm{f} \\
\mathrm{m}\end{array}$ & $\begin{array}{l}\text { décharge } \\
\text { fluide } \\
\text { minimum }\end{array}$ & \\
\hline$\underset{\mathrm{m}}{\mathrm{J} \mathrm{kg}^{-1} \mathrm{~K}^{-1}}$ & $\begin{array}{l}\max \\
\mathrm{s} \\
\mathrm{t}\end{array}$ & $\begin{array}{l}\text { maximum } \\
\text { solide } \\
\text { total. }\end{array}$ & \\
\hline$\underset{\substack{\mathrm{W} \\
\mathbf{W}}}{\mathrm{K}}$ & & & \\
\hline
\end{tabular}
$\begin{array}{cc}\mathrm{K} & \text { 1. Introduction. }- \text { Le problème du stockage de } \\ \mathrm{K}^{-1} & \text { l'énergie se pose dès qu'il faut adapter le niveau d'une }\end{array}$ $\mathrm{K}$ production à celui d'une demande. Les solutions $\mathrm{J} \quad$ apportées à ce problème sont très spécifiques de la $\mathrm{m}^{3} \quad$ forme d'énergie considérée (accumulateurs pour l'énerJ gie électrique, volant d'inertie pour l'énergie mécanique, etc...). 
L'intérêt croissant porté à l'énergie solaire a conduit à étudier des stockages d'énergie thermique puisque c'est sous cette forme que la majeure partie de l'énergie solaire captée actuellement est transformée tant pour les besoins de l'habitat que pour les besoins de puissance.

Le niveau thermique requis pour l'habitat et pour certaines applications thermodynamiques à basse température $\left(<100^{\circ} \mathrm{C}\right)$ a imposé l'eau comme milieu de stockage [1]. Les lits de pierres ont aussi été expérimentés pour ces applications en utilisant soit l'eau [2], soit l'air [3], [4] comme fluide caloporteur.

La conception de stockage faiblement pressurisés utilisables dans des centrales solaires à concentration dont le niveau thermique maximum est de l'ordre de $300^{\circ} \mathrm{C}$ a conduit à utiliser des fluides organiques à point d'ébullition élevé comme milieu de stockage [5], [6]. Ces fluides étant d'un coût élevé, il est intéressant de minimiser la quantité de fluide utilisée par unité de volume de milieu de stockage.

Cet objectif est atteint en utilisant des stockages composites fluide/solides dont le dimensionnement et les performances sont étudiés ici.

2. Stockages fluide/solides. -2.1 CONCEPTION GÉNÉRALE (voir Fig. 1). - Un tel stockage se compose d'une enceinte contenant un empilement de solides disposés régulièrement ou en vrac, de tuyauteries d'entrée/sortie assurant la circulation du fluide caloporteur suivant la direction verticale.

La taille moyenne des solides et la porosité de l'empilement imposent la surface volumique de contact entre les solides et le fluide qui occupe tout le volume qui lui est offert.

Les valeurs des capacités calorifiques du fluide et des solides $\left(\rho_{\mathrm{f}} C_{\mathrm{f}}\right.$ et $\left.\rho_{\mathrm{s}} C_{\mathrm{s}}\right)$ étant du même ordre de

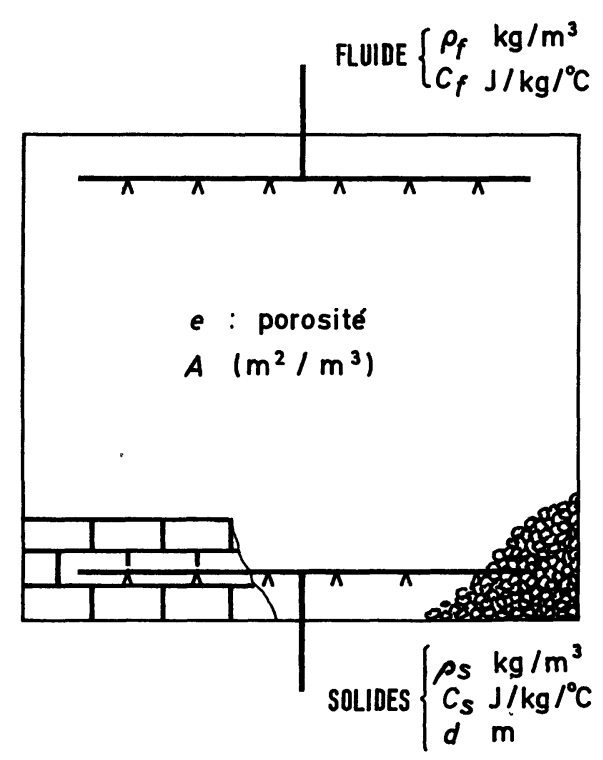

Fig. 1. - Conception d'un stockage fluide/solides.

[Design of a fluid/solids storage unit.] grandeur $\left(\simeq 2 \times 10^{6} \mathrm{~J} \mathrm{~m}^{-3} \mathrm{~K}^{-1}\right)$, la substitution d'un volume de solides à un même volume de liquide ne modifie pas de manière notable la valeur de la densité d'énergie du milieu de stockage fluide/solides par rapport à celle du fluide seul.

Durant les périodes de charge du stockage, le fluide chaud est introduit à la partie supérieure de l'enceinte, cède sa chaleur aux solides, et ressort froid à la partie inférieure. Le sens de circulation est inversé lors des périodes de décharge, les solides cédant alors leur chaleur au fluide qui entre froid à la partie inférieure de l'enceinte et sort chaud à la partie supérieure.

Le respect du sens de circulation lors de ces opérations conditionne la bonne stratification des températures dans le milieu de stockage [7].

2.2 MODÉLISATION DES TRANSFERTS THERMIQUES. L'isolation du stockage est supposée suffisante pour que les pertes thermiques latérales du stockage soient négligeables durant les périodes de transfert thermique entre les solides et le fluide (charge et décharge).

Moyennant certaines précautions quant à la conception des tuyauteries d'entrée/sortie, l'hypothèse d'une répartition uniforme des vitesses du fluide dans une section horizontale du stockage est vérifiée expérimentalement [7].

Nous considérons en outre que les caractéristiques physiques du fluide et des solides sont constantes ainsi que le coefficient d'échange $h$ caractérisant le transfert entre les deux phases. De plus, le.nombre de Biot $h d / \lambda_{\mathrm{s}}$ est suffisamment faible pour que la température d'un solide quelconque puisse être considérée comme uniforme.

Ces hypothèses conduisent à la représentation du stockage à l'aide d'un modèle monodimensionnel (voir Fig. 2) décrit par les équations (1) et (2).

$$
\left\{\begin{array}{l}
e \rho_{\mathrm{f}} C_{\mathrm{f}} \frac{\partial F}{\partial t}+e v \rho_{\mathrm{f}} C_{\mathrm{f}} \frac{\partial F}{\partial x}=-h A(F-S) \\
(1-e) \rho_{\mathrm{s}} C_{\mathrm{s}} \frac{\partial S}{\partial t}=-h A(S-F) .
\end{array}\right.
$$

En utilisant les coefficients $a$ et $b$ définis par les relations (3) et (4)

$$
\begin{aligned}
& a=\frac{h A}{e \rho_{\mathrm{f}} C_{\mathrm{f}}} \\
& b=\frac{e \rho_{\mathrm{f}} C_{\mathrm{f}}}{(1-e) \rho_{\mathrm{s}} C_{\mathrm{s}}}
\end{aligned}
$$

et en appliquant le changement de variable suivant :

$$
\begin{aligned}
& X=a x / v \\
& Z=a b\left(t-\frac{x}{v}\right)
\end{aligned}
$$


TEMPERATURES à l'INSTANT : $\boldsymbol{t}$

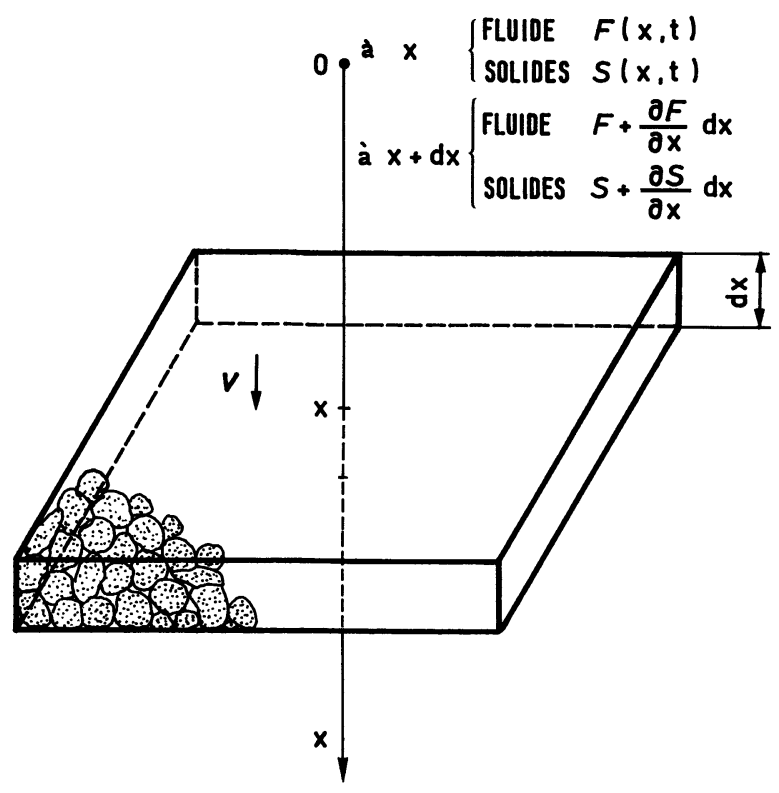

Fig. 2. - Modèle mathématique.

[Mathematical model.] le système d'équations initial s'écrit sous la forme simplifiée :

$$
\left\{\begin{array}{l}
\frac{\partial F}{\partial X}=S-F \\
\frac{\partial S}{\partial Z}=F-S .
\end{array}\right.
$$

Ces deux systèmes d'équations équivalents ont été étudiés par de nombreux auteurs en admettant diverses conditions :

- Stockage initialement à température uniforme $F_{0}$, saut de température du fluide d'amplitude $F_{1}-F_{0}$ à une des extrémités, débit constant [8], [9], [10].

- Profil initial de température du stockage fixé, température du fluide à une des extrémités du stockage donnée en fonction du temps, débit constant [11].

Les solutions proposées sont données sous forme de séries et d'intégrales qui sont en général lourdes à utiliser.

Une excellente approximation de la solution correspondant au premier cas a été donnée par Klinkenberg [12], [13] sous la forme suivante :

$$
\begin{aligned}
& \frac{F(X, Z)-F_{0}}{F_{1}-F_{0}}=\frac{1}{2}\left[1+\operatorname{erf}\left(\sqrt{Z}-\sqrt{X}+\frac{1}{8 \sqrt{Z}}+\frac{1}{8 \sqrt{X}}\right)\right] \\
& \frac{S(X, Z)-F_{0}}{F_{1}-F_{0}}=\frac{1}{2}\left[1+\operatorname{erf}\left(\sqrt{Z}-\sqrt{X}-\frac{1}{8 \sqrt{Z}}-\frac{1}{8 \sqrt{X}}\right)\right] .
\end{aligned}
$$

Cette solution, d'un maniement aisé, requiert simplement une tabulation de la fonction d'erreur, donc des moyens de calcul limités.

Le théorème de Duhamel permet d'étendre cette solution au cas où la température à une des extrémités du stockage varie en fonction du temps [7].

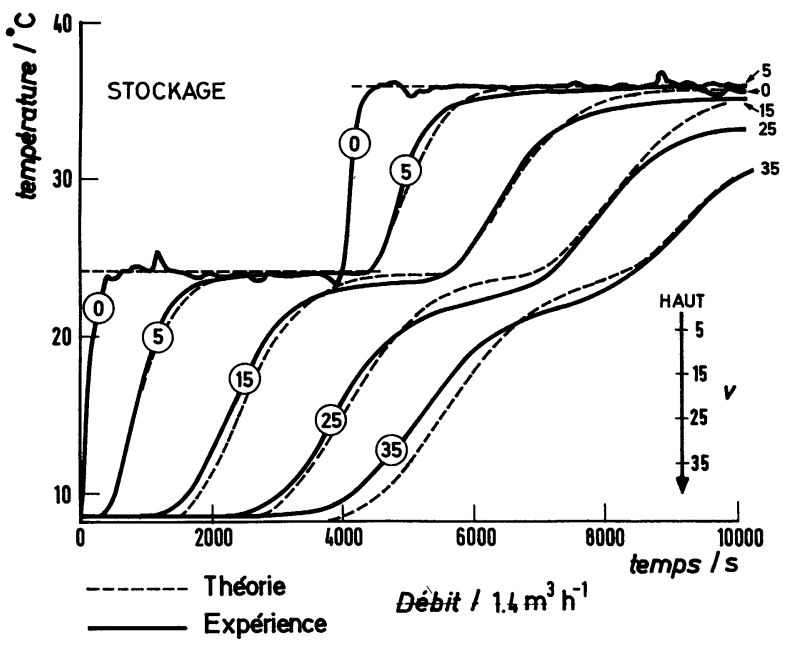

Fig. 3. - Vérification expérimentale du modèle mathématique [7]

[Experimental verification of the mathematical model [7].]
Nous obtenons ainsi un instrument de calcul rapide pour recaler les résultats expérimentaux. Malgré sa relative simplicité, ce modèle permet de prévoir d'une manière suffisamment précise le comportement d'une unité expérimentale de stockage fluide/solides (voir Fig. 3).

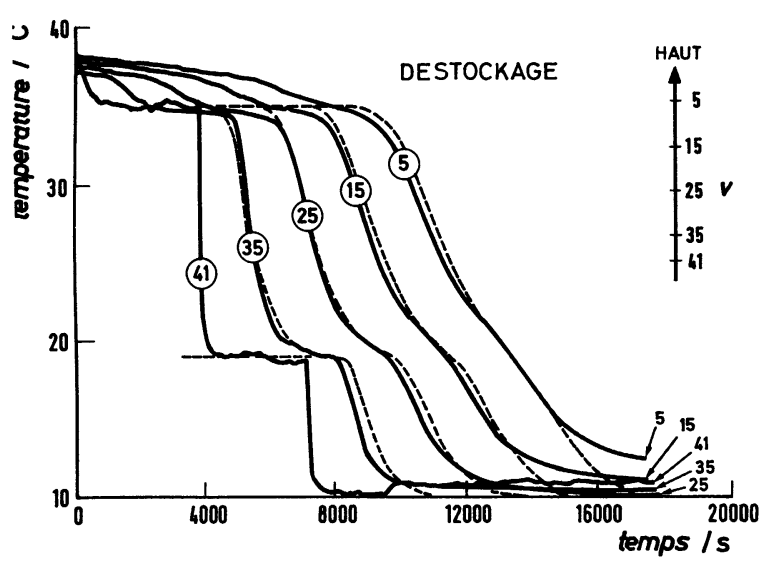


Moyennant quelques hypothèses supplémentaires [7], on peut obtenir un meilleur accord avec l'expérience mais ce, au détriment de la simplicité d'utilisation des expressions (9) et (10). L'étude de l'influence des divers paramètres est aussi facilitée par ces relations.

3. Dimensionnement. - 3.1 PosITION DU PROBLÈME. - L'utilisateur a besoin pendant un temps $t_{\mathrm{d}}$ d'une puissance thermique $P$ à un niveau de température $F_{1}$; le fluide utilisé retournant au stockage à la température $F_{0}$. La température $F_{1}$ peut subir une légère baisse définie comme un pourcentage $\delta$ de l'écart $F_{1}-F_{0}$. L'isolation du stockage est suffisante pour que les pertes thermiques durant la décharge soient négligeables.

Si le front de température dans le stockage restait plat pendant la décharge le volume du stockage ainsi que le diamètre de l'orthocylindre correspondant seraient donnés par :

$V_{\mathrm{m}}=\frac{\pi D_{\mathrm{m}}^{3}}{4}=\frac{P t_{\mathrm{d}}}{\left(e \rho_{\mathrm{f}} C_{\mathrm{f}}+(1-e) \rho_{\mathrm{s}} C_{\mathrm{s}}\right)\left(F_{1}-F_{0}\right)}$.

Comme le front s'étale au cours du temps, nous devons prévoir un volume supplémentaire.

3.2 LARGEUR DU FRONT DE TEMPÉRATURE. - L'évaluation des coefficients $a$ et $b$ est possible dès que l'on a choisi une corrélation permettant le calcul du coefficient d'échange $h$ [14], [15], [16].

Le tracé du profil de la température du fluide dans le stockage à un instant $t$ fait apparaître trois zones (Fig. 4).

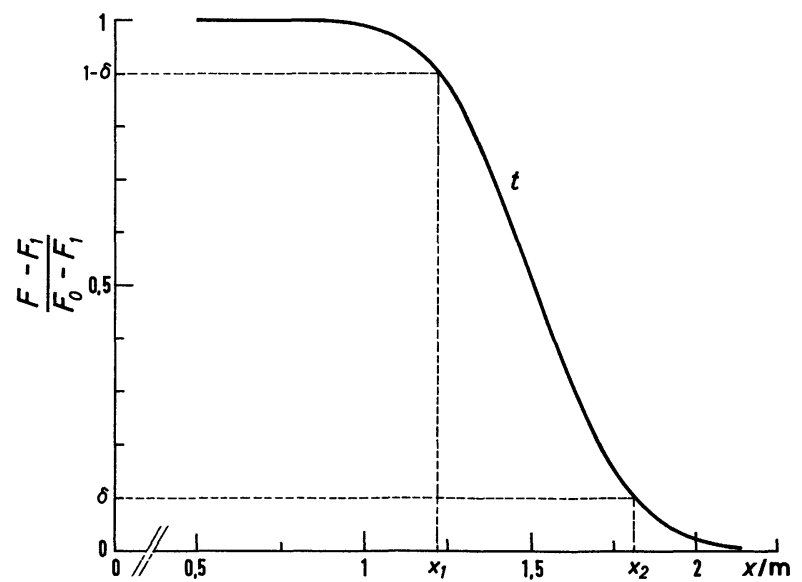

Fig. 4. - Largeur du front de température : La courbe est tracée pour les valeurs $a=0,01 \mathrm{~s}^{-1}, b=0,51, v=4,1 \times 10^{-4} \mathrm{~m} / \mathrm{s}$, à l'instant $t=3 \mathrm{~h}$. Ceci correspond à un stockage devant assurer une puissance de $25 \mathrm{~kW}$, pendant $3 \mathrm{~h}$ avec $F_{1}-F_{0}=50^{\circ} \mathrm{C}$, $e=0,35$ et $d=2 \mathrm{~cm}$.

[Width of the thermocline : This plot is made taking $a=0.01 \mathrm{~s}^{-1}$ $b=0.51, v=4.1 \times 10^{-4} \mathrm{~m} / \mathrm{s}$ at $t=3 \mathrm{~h}$; these values correspond to a storage unit capable of delivering $25 \mathrm{~kW}$ during 3 hours with $F_{1}-F_{0}=50^{\circ} \mathrm{C}, e=0.35, d=2 \mathrm{~cm}$.] $-0<x<x_{1}$ la température du stockage est $F_{0}$ à $\delta\left(F_{1}-F_{0}\right)^{\circ} \mathrm{C}$ près.

$-x_{1} \leqslant x \leqslant x_{2}$ zone de transition.

$-x_{2}<x$ la température du stockage est $F_{1}$ à $\delta\left(F_{1}-F_{0}\right)^{\circ} \mathrm{C}$ près.

La largeur $\Delta x=x_{2}-x_{1}$ de la zone de transition est appelée largeur du front de température.

A partir de la relation (9), on trouve [17] :

$$
\Delta x=4 \beta \frac{\sqrt{b}}{(b+1)^{3 / 2}} \frac{v}{\sqrt{a}} \sqrt{t}
$$

avec

$$
\operatorname{erf} \beta=1-2 \delta
$$

3.3 CALCUl DU VOlUME SUPPlÉMENTAIRE, EFFICACITÉ DE RÉCUPÉRATION. - Afin d'assurer la décharge du stockage au niveau de température $F_{1}$ à $\delta\left(F_{1}-F_{0}\right)^{\circ} \mathrm{C}$ près, il est nécessaire que la hauteur minimale $D_{\mathrm{m}}$ soit augmentée de la moitié de la largeur du front à l'instant $t_{\mathrm{d}}$ de fin de décharge (Fig. 5).

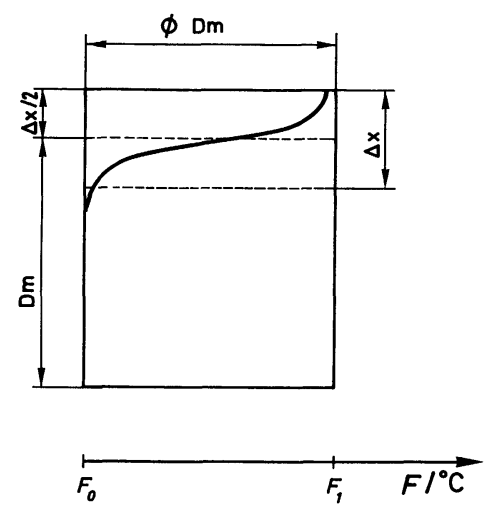

Fig. 5. - Evaluation du volume supplémentaire.

[Evaluation of the additional volume.]

Le volume supplémentaire ainsi introduit correspond à une perte d'énergie non récupérable au niveau de température désiré. Nous pouvons alors définir une efficacité de récupération

Energie déstockable au niveau

$$
\begin{gathered}
\varepsilon_{1}=\frac{\text { de température souhaitée }}{\text { Energie stockée }} \\
\varepsilon_{1}=\frac{1}{1+\frac{\Delta x}{2 D_{\mathrm{m}}}}
\end{gathered}
$$

Pour $P=25 \mathrm{~kW}, \quad F_{1}-F_{0}=50^{\circ} \mathrm{C}, t_{\mathrm{d}}=3 \mathrm{~h}$, $\delta=0,1, e=0,35, d=2 \mathrm{~cm}$, on trouve $\varepsilon_{1}=0,83$.

Le milieu de stockage est d'autant mieux utilisé que l'efficacité de récupération est plus élevée (ou que le volume supplémentaire est petit). L'étude de 
l'influence des différents paramètres montre que ceci est réalisé pour un stockage de taille donnée quand :

- le diamètre des particules solide décroît,

- la hauteur du stockage croît jusqu'à la hauteur de l'orthocylindre, le gain pour de plus grandes hauteurs restant faible,

- le temps de décharge croît; il faut noter ici que le modèle utilisé ne tient compte ni des pertes thermiques ni de la conduction axiale dont les effets peuvent ne plus être négligeables si la durée de décharge devient très longue.

On peut montrer en outre que plus la taille du stockage est importante plus il est performant (meilleure efficacité de récupération).

3.4 Comparaison aVeC UN STOCKaGe EN FLUIDE SEUL. - Les calculs effectués pour le stockage fluide/solides peuvent être repris pour le cas d'un stockage en fluide seul, en considérant que la conduction est le seul mécanisme d'étalement du front de température [17].

Pour un même service rendu, le volume supplémentaire est plus faible dans le cas d'un stockage en fluide seul et par conséquent le coefficient de récupération est plus élevé que dans le cas d'un stockage fluide/solides.

Néanmoins, l'économie de fluide dans le stockage fluide/solides reste de l'ordre de $60 \%$ par rapport au stockage en fluide seul.

4. Etude thermodynamique. - Le transfert de chaleur entre les solides et le fluide est une cause d'irréversibilités dont nous allons évaluer l'importance sur les performances du stockage.

4.1 Pertes PAR IRRÉVersibilités. - Considérons le système constitué par un stockage fluide/solides, les seuls échanges avec le milieu extérieur étant les échanges de masse à l'entrée et à la sortie du stockage.

Dans ce cas, la variation totale d'entropie (entropie du système plus entropie du milieu extérieur) se réduit à la production d'entropie à l'intérieur du système qui est due à l'échange de chaleur entre les phases fluide et solides à travers un écart fini de température. Par conséquent :

$$
\frac{\mathrm{d} S_{\mathrm{t}}^{*}}{\mathrm{~d} t}=\int_{0}^{l} \underbrace{h A s \frac{(F-S)}{S} \mathrm{~d} x}_{\begin{array}{c}
\text { entropie } \\
\text { des solides }
\end{array}}+\int_{0}^{l} \underbrace{h A s \frac{(S-F)}{F} \mathrm{~d} x}_{\begin{array}{c}
\text { entropie } \\
\text { du fluide }
\end{array}}
$$

soit

$$
\Delta S_{\mathrm{t}}^{*}=\int_{0}^{t} \int_{0}^{l} a e \rho_{\mathrm{f}} C_{\mathrm{f}} s\left(\frac{F}{S}+\frac{S}{F}-2\right) \mathrm{d} x \mathrm{~d} t .
$$

Si $T_{0}$ est la température du milieu de référence, $T_{0} \Delta S_{t}^{*}$ représente les pertes par irréversibilité au cours d'une charge ou d'une décharge. La minimisation de ces pertes conduit à des conclusions identiques à celles énoncées en 3.3.

4.2 TRAVAil MaXimum RÉCUPéRABLE. - Le travail maximum récupérable en refroidissant un volume $V$ de fluide de la température $F_{1}$ à la température $F_{0}$ est égal à la variation d'exergie.

$$
\begin{aligned}
W_{\max } & =\Delta\left(U-T_{0} S^{*}\right) \\
& =V \rho_{\mathrm{f}} C_{\mathrm{f}}\left(F_{1}-F_{0}\right) \eta\left(F_{1}\right) \\
\text { avec } \quad \eta\left(F_{1}\right) & =1-\frac{T_{0}}{\left(F_{1}-F_{0}\right)} \ln \left(\frac{F_{1}}{F_{0}}\right) .
\end{aligned}
$$

Par conséquent, le travail maximum récupérable $W_{\mathrm{d}}$ au cours d'une décharge est (Fig. 6)

$$
W_{\mathrm{d}}(t)=\int_{0}^{t} Q \rho_{\mathrm{f}} C_{\mathrm{f}}\left(F(l, t)-F_{0}\right) \eta(F(l, t)) \mathrm{d} t .
$$

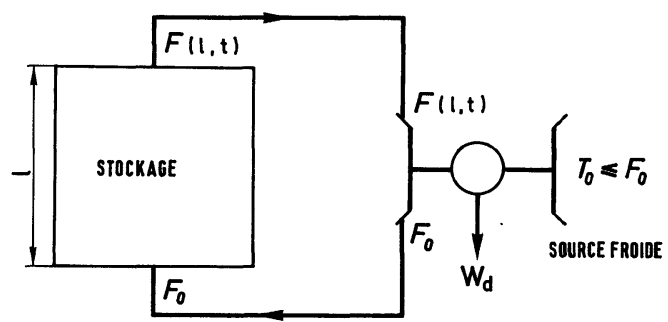

Fig. 6. - Production de travail à partir du stockage.

[Work production from storage.]

Si la décharge est arrêtée à l'instant $t_{\mathrm{d}}$ tel que $F\left(l, t_{\mathrm{d}}\right)=F_{1}-\delta\left(F_{1}-F_{0}\right)$, nous pouvons définir un coefficient de récupération

$$
\varepsilon_{2}=\frac{W_{\mathrm{d}}\left(t_{\mathrm{d}}\right)}{W_{\mathrm{d}}(\infty)}
$$

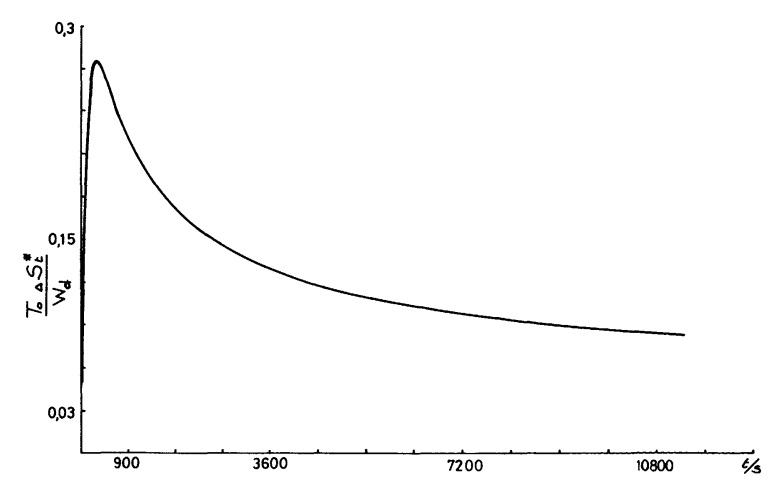

Fig. 7. - Evolution des pertes par irréversibilités. La courbe est tracée pour le même cas que celui de la figure 4. $T_{0}=F_{0}=523 \mathrm{~K}$.

[Evolution of the loss by irreversible processes. The plot is made for the same case as that of figure 4. $T_{0}=F_{0}=523 \mathrm{~K}$.] 
Pour les mêmes valeurs numériques que celles du paragraphe 3.3 et avec $T_{0}=300 \mathrm{~K}$ on trouve $\varepsilon_{2}=0,82$.

Les pertes par irréversibilité croissent d'abord plus vite que le travail récupéré à la décharge à cause du saut de température à l'entrée du stockage (Fig. 7), puis le rapport $\frac{T_{0} \Delta S_{\mathrm{t}}^{*}}{W_{\mathrm{d}}}$ décroît rapidement et les pertes ne représentent plus qu'une part du travail d'autant plus faible que $T_{0}$ l'est.

5. Conclusion. - La méthode de dimensionnement proposée est fondée sur un modèle simple dont la validité a pu être vérifiée expérimentalement dans le cas de l'eau circulant à travers un lit de pierres [4], et qui est en cours de test pour un stockage fonctionnant à plus haute température $\left(300^{\circ} \mathrm{C}\right)$.

Les pertes par irréversibilités qui sont propres à ce type de stockage ne condamnent pas le principe et l'économie de fluide reste substantielle.

Les pertes de charge dues à la circulation du fluide à travers les solides restent négligeables du fait des faibles vitesses interstitielles.

Avec l'élévation de température se pose le problème du frettage de l'empilement dans l'enceinte qui peut amener à des épaisseurs de parois importantes.

Certains problèmes technologiques restent donc à résoudre mais la possibilité d'emploi de résidus solides industriels compatibles avec les fluides envisagés permet de penser que le coût spécifique de telles installations sera compétitif.

\section{Bibliographie}

[1] Ployart, R., Torrenti, R., E.D.F., Information sur l'énergie, J. 36N (décembre 1978).

[2] Bernard, J., SAlagnaC, J.-L., E.D.F., Information sur l'énergie, J. 36N (décembre 1978).

[3] Klein, S. A., Beckman, W. A., Duffie, J. A., Sol. Energy 19 (1977) 509-512.

[4] Salagnac, J.-L., Thermal insulation laboratory Technical University of Denmark (mai 1975).

[5] De Moncuit, M., A.I.M., Liège, Energie Solaire (1977).

[6] Peube, J.-L., D'Utruy, B., Blay, D., Entropie 85 (1979) 48-54.

[7] Bernard, J., Salagnac, J.-L., CEA/SEEN-RT-78-147 (mai 1978).

[8] SchumanN, T. E. W., J. Franklin Inst. 208 (1929) 405-416.
[9] HAUSEN, H., Warmeübertragung im Gegenstrom, Gleichstrom und Kreuzstrom (Springer Verlag, Berlin) (1976), p. 259.

[10] Carslaw, H. S., Jaeger, J. C., Conduction of Heat in Solids (Clarendon Press, Oxford) (1959), p. 391.

[11] Kohlmayr, G. F., J. Heat Transfer 2 (1968) pp. 176-178

[12] Klinkenberg, A., Ind. Eng. Chem. 40 (1948) pp. 1992-1994.

[13] Klinkenberg, A., Ind. Eng. Chem. 46 (1954) 2285-2289.

[14] Ranz, W. E., Chem. Eng. Prog. 48 no 5 (1952) 247-253.

[15] JАKOB, M., Heat Transfer (John Wiley and Sons, N.Y.) II (1957) 261.

[16] GunN, D. J., Int. J. Heat Mass Transfer 21 (1978) 467-476.

[17] Ayoub, J. A., C.E.A./SEEN-RT-79-235 (juin 1979). 